\title{
Ordenando el caos: cuatro enfoques metodológicos para investigar en políticas públicas
}

\author{
Sorting out chaos: Four methodological approaches \\ to do research in public policy
}

\author{
ALEJANDRO HERNÁNDEZ-LUIS
}

Facultad Latinoamericana de Ciencias Sociales. FLACSO-Ecuador

CAMILA CARRASCO

Centro de Políticas Públicas. Universidad Católica de Temuco

SARAI GARCÍA-GUERRA

Facultad Latinoamericana de Ciencias Sociales. FLACSO-Ecuador

\section{Cómo citar/Citation}

Hernández-Luis, A., Carrasco, C. y García-Guerra, S. (2021). Ordenando el caos: cuatro enfoques metodológicos para investigar en políticas públicas. Revista Española de Ciencia Política, 56, 173-196. Doi: https://doi.org/10.21308/recp.56.07

\section{Resumen}

El análisis de políticas públicas es un campo disciplinar donde convergen múltiples teorías y procedimientos metodológicos. Esta nota de investigación aborda la pregunta sobre si es posible ordenar el conocimiento de manera que los supuestos filosóficos, marcos analíticos y métodos puedan ser alineados. Para ello, utilizamos la tipología propuesta por Patrick Jackson como esquema organizacional de los debates en filosofía de la ciencia. Sobre esa base, proponemos una distribución de las teorías macro del análisis de políticas en correspondencia con las cuatro metodologías definidas en el esquema: neopositivismo, realismo, analiticismo y reflexivismo. El propósito del ejercicio es hacer que las propuestas teóricas que conforman el análisis de políticas sean más accesibles para los investigadores y, de esta forma, lograr que se destaquen los elementos de distinción y las implicaciones prácticas para las metodologías de investigación. Lo anterior permitirá visualizar los posibles caminos que consideramos que pueden conducir a realizar diseños de investigación más consistentes, coherentes y congruentes.

Palabras clave: políticas públicas, ontología, metodología, marcos analíticos.

\begin{abstract}
Public policy analysis (PPA) is a disciplinary field where multiple theories and methodological procedures converge. This research note addresses whether it is possible to organize knowledge
\end{abstract}


in a way that philosophical assumptions, analytical frameworks and methods could be aligned. To this aim, Patrick Jackson's typology is used as an organizational scheme of the debates in philosophy of science. Based on this, we propose a distribution of PPA macro theories in correspondence with the four methodologies defined in Jackson's scheme: neopositivism, realism, analyticism and reflexivity. The purpose of this exercise is to make PPA theoretical proposals more accessible for researchers and, this way, to highlight their distinctive elements and practical implications for research methodologies. This will allow to visualize the potential paths that could lead towards more consistent, coherent and congruent research designs.

Keywords: public policy, ontology, methodology, analytical frameworks.

\section{INTRODUCCIÓN: LA INVESTIGACIÓN DE LAS POLÍTICAS PÚBLICAS EN AMÉRICA LATINA}

El análisis de políticas es un campo joven en la temporalidad del desarrollo científico, que comenzó a configurarse en los Estados Unidos apenas a inicios de la década de 1950 (Lasswell, 1951). Como en todo campo de estudio, se han propuesto diversas teorías en función de las preferencias de los investigadores a lo largo de los años. Estas se derivan de su comprensión del mundo y de la ciencia o por efectos de difusión o adscripción a determinada escuela. Dada la diversidad de enfoques en la actualidad, el análisis de políticas es visto como un espacio desordenado donde confluyen múltiples marcos, teorías, enfoques, modelos y metodologías. Este caos suele ser una de las causas que abruman a los investigadores jóvenes que comienzan a acercarse al objeto de estudio y, sobre todo, a los practicantes, quienes no suelen contar con el tiempo para adentrarse en debates abstractos.

En América Latina, la historia del campo de estudio tiene una complejidad añadida. El concepto políticas públicas y su lenguaje asociado irrumpieron en la región a finales de la década de 1980 de la mano del Consenso de Washington y los organismos multilaterales de financiamiento (Roth, 2014). El concepto, además, vino acompañado de la nueva gestión pública y las pretensiones de modernización y mejora de la Administración pública (Peters y Pierre, 2005). Lo complejo de esta historia se debe al menos a tres factores: en primer lugar, el desarrollo del campo no se originó en la región y llegó de manera tardía. En segundo lugar, está el hecho de la asociación del lenguaje y sus conceptos fundamentales al neoliberalismo, debido al momento y la forma de su inclusión en la Administración pública latinoamericana (apreciación sesgada de la disciplina que causa serios inconvenientes). Y, finalmente, el problema de la convivencia de distintos paradigmas, lenguajes y tradiciones de la Administración pública que se explican a continuación.

Contrariamente a las pretensiones de los organismos multilaterales de financiamiento (Aguilar, 2007), el lenguaje de las políticas públicas no llegó a América Latina a manera de borrón y cuenta nueva. El espacio en el cual entró se caracterizaba - y aún se caracteriza - por una construcción híbrida entre tradiciones heterogéneas, 
como la heredada de la colonia, la planificación soviética y el desarrollismo cepalino; y también por un patrón de relaciones sociales marcado por la personalización del poder (Méndez, 1999) y el consecuente clientelismo. Esta confluencia perversa es responsable de las constantes confusiones, estiramientos conceptuales, ambigüedades e incorrectas homologaciones que existen en la terminología que manejan los Gobiernos latinoamericanos y que, en ocasiones, también pueden encontrarse en publicaciones académicas e investigaciones desarrolladas por equipos de trabajo de organizaciones internacionales y no gubernamentales. Entre los principales problemas que al respecto pueden señalarse están: el uso indistinto de los términos política, programa, plan y proyecto, sin una clara diferenciación de sus características y alcances; la idea de que las políticas públicas, para ser tales, tienen que estar escritas en un documento aprobado por un ente rector (ministerios o secretarías) y que se denomine «la política de...», o la necesidad de que exista una ley $-\mathrm{y}$ no otro u otros instrumentos de autoridad - para poder afirmar que existe una política pública.

El panorama descrito hasta aquí es desalentador. La descripción es necesaria, sin embargo, no para recargar de pesimismo a una situación ya compleja, sino para conocer las particularidades del campo en el contexto latinoamericano y, a partir de ahí, pensar y plantear caminos a través de los cuales avanzar. Uno de estos es la organización del estado del conocimiento. Este tipo de esfuerzos se ha realizado anteriormente, siendo el libro editado por Paul Sabatier (2010) el más conocido en la región, sobre todo después de la traducción que realizó la Jefatura de Gabinete de Ministros de Argentina. Otros autores como Roth (2014) y Fontaine (2015), también han publicado manuales que tienen el objetivo de describir y explicar las distintas teorías del área. Estos últimos añaden un importante esfuerzo de contextualización, explicando los distintos enfoques, así como las problemáticas de la inserción, traducción y estudio en la región.

El presente trabajo no pretende tener el mismo alcance que los textos mencionados anteriormente. Su objetivo no es explicar las particularidades de cada una de las teorías, sino que nos preguntamos si es posible ordenar el conocimiento de manera que los investigadores puedan contar con una orientación que les permita alinear supuestos ontológicos, teorías y métodos. Es decir, pretendemos contribuir a una reflexión que destaque la importancia de definir compromisos ontológicos dentro de la investigación y, en función a ello, adoptar teorías y métodos coherentes con esos compromisos. De esta manera, consideramos que para dar cuenta de un resultado de política o anticipar los efectos de la toma de decisión de forma adecuada, las investigaciones deben ser transparentes respecto a sus apuestas filosóficas y propuestas epistemológicas.

Para ello, presentaremos el esquema organizacional desarrollado por Jackson (2016: 36) a manera de tipología, que fue formulado con el objetivo de hacer que las reflexiones sistemáticas encontradas en la filosofía de la ciencia sean accesibles para los investigadores. Después de mostrar la tipología en su planteamiento original, mostraremos cuál es su utilidad para organizar las teorías del análisis de políticas no de una manera exhaustiva, sino como una forma de generar categorías que contribuyan a aclarar las diversas discusiones y que ayuden a los investigadores a comprender las implicaciones prácticas de utilizar esas teorías y métodos para sus estudios. 


\section{UNA TIPOLOGÍA PARA LA ORGANIZACIÓN DE CAMPOS DISCIPLINARES}

La preocupación de Jackson (id.) está relacionada con el problema de que la filosofía de la ciencia no tiene un esquema organizacional ampliamente aceptado, que divida autores y posiciones en distintas escuelas de pensamiento. Las discusiones generalmente son muy dispersas y comprender las disputas entre los filósofos de la ciencia es un asunto en extremo complicado. Por ejemplo, las frecuentes distinciones entre positivismo e interpretativismo, así como entre cuantitativo y cualitativo suelen basarse en una diferencia de método en lugar de una diferencia de metodología. Realizadas así, estas distinciones pueden ser demasiado simplistas ${ }^{1}$.

Ante esta problemática, Jackson (id.) plantea una tipología que pretende mostrar diferentes posiciones dentro de la filosofía de la ciencia y, a partir de ahí, aclarar las implicaciones que una determinada combinación de compromisos ontológicos tiene en la realidad práctica de la producción de conocimiento. La tipología se plantea en un formato dos por dos, donde se combinan ontologías filosóficas por un eje y ontologías científicas por el otro. El producto de la combinación son las cuatro metodologías que aparecen en la tabla siguiente: neopositivismo, analiticismo, reflexivismo y realismo crítico.

TABLA 1.

Tipología de CUATRO METODOlOGías

\begin{tabular}{|c|c|c|c|}
\hline & & \multicolumn{2}{|c|}{$\begin{array}{l}\text { Relación entre conocimiento y observación } \\
\text { (ontologías científicas) }\end{array}$} \\
\hline & & Fenomenalismo & Transfactualismo \\
\hline \multirow{2}{*}{$\begin{array}{l}\text { Relación entre el } \\
\text { conocedor y lo conocido } \\
\text { (ontologías filosóficas) }\end{array}$} & $\begin{array}{c}\text { Dualismo } \\
\text { mente mundo }\end{array}$ & Neopositivismo & Realismo crítico \\
\hline & $\begin{array}{c}\text { Monismo } \\
\text { mente mundo }\end{array}$ & Analiticismo & Reflexivismo \\
\hline
\end{tabular}

Fuente: Jackson (2016).

Ahora bien, aquí son necesarias dos aclaraciones: en primer lugar, el significado de cada uno de los elementos de la tipología y la coherencia interna del esquema; en segundo lugar, el alcance y la intención de esta, sobre todo porque coincide con el trabajo de alineación con las teorías de las políticas públicas que se realizará más adelante.

En relación con la primera cuestión, se parte de la idea de que una teoría o una investigación siempre debe localizar y especificar su posición respecto de, al menos, tres cosas: el investigador, el mundo que se investiga y el carácter de la relación entre estos. La

1. Por ejemplo, King et al. (2000: 39-41) asignan a las investigaciones que realizan entrevistas en profundidad u observaciones participantes la función de productoras de información para comprobar la teoría elaborada a partir de datos brutos o manejo de variables. Con ello, las investigaciones que utilizan métodos cualitativos quedan simplemente relegadas a un segundo plano. 
relación entre el investigador y el mundo (ontología filosófica) suele darse de dos formas distintas: a) dualismo mente-mundo (separación entre el investigador y el mundo, de manera que el conocimiento válido debe estar relacionado con una correspondencia precisa entre proposiciones empíricas y teóricas), y b) monismo mente-mundo (el investigador es parte del mundo, por lo que el mundo es endógeno a las prácticas sociales de producción del conocimiento y, por lo tanto, no es una simple descripción de objetos mundanos ya existentes). Esta dicotomía ha sido frecuentemente caracterizada como positivista para referirse al dualismo (Wendt, 1999) e interpretativista para señalar al monismo (Yanow y Schwartz-Shea, 2006). Sin embargo, esta distinción es poco útil, ya que no aclara suficientemente todos los problemas en juego de la distinción filosófica.

La necesidad de esclarecer las categorías para ordenar los debates contemporáneos sobre investigación social llevó a Jackson a plantear la división entre dos tipos de ontologías científicas: fenomenalismo y transfactualismo (2016: 41). La cuestión clave de esta división es determinar si el conocimiento se produce a través de cosas que pueden experimentarse y observarse empíricamente (fenomenalismo) o si es posible generar conocimiento de objetos que son inobservables en principio (transfactualismo). La idea es que tanto en la postura monista como dualista los investigadores pueden analizar solamente hechos/fenómenos o ir más allá de esos hechos para comprender los procesos y factores más profundos que los generan.

La combinación de las dos ontologías, como mencionamos, produce cuatro metodologías. Cabe señalar que el término metodología en este trabajo no es entendido como el conjunto de métodos utilizados en una investigación científica, sino como «una preocupación con la estructura lógica y el procedimiento de la investigación científica» (Sartori, 1970: 1033). Estas metodologías tienen un conjunto de elementos característicos.

El neopositivismo se originó en la filosofía empiricista y el positivismo lógico. Su objetivo es producir leyes de cobertura, por lo que se privilegian explicaciones orientadas por variables que prueben regularidades entre un explanans $(\mathrm{X}) \mathrm{y}$ un explanandum $(\mathrm{Y})$. Para ello se utilizan estadísticas, contrafácticos y ensayos aleatorios controlados, entre otros. Sus compromisos ontológicos se asocian a una realidad objetiva y su epistemología empiricista es firmemente antimetafísica. El realismo crítico es parte de las filosofías de la ciencia pospositivista. Busca explicar las causas genuinas de los fenómenos de manera distinta al neopositivismo. En este sentido, sus investigaciones prefieren determinar fuerzas o mecanismos que proporcionen explicaciones causales de rango medio, usualmente construidas a través de comparaciones de $n$ pequeńa o estudios de caso a profundidad. Si bien sus compromisos ontológicos también se asocian a una realidad objetiva, su epistemología no se detiene en lo directamente observable. El analiticismo y el reflexivismo se originaron en las tradiciones filosóficas idealistas y otras no empiricistas. Ambas metodologías rechazan la idea de que exista una realidad independiente de la mente. Sin embargo, el analiticismo, al ser consistente con los enfoques constructivistas sociales, entiende que las teorías son idealizaciones y simplificaciones del mundo, por lo que el esfuerzo científico implica desagregar y simplificar los fenómenos a través de tipos ideales para su mejor comprensión. Por su parte, el reflexivismo incorpora 
enfoques activistas o comprometidos, en donde la investigación científica está relacionada dialécticamente con las condiciones sociales de su producción y conduce a una crítica sobre los supuestos opresivos de las relaciones sociales (McArthur, 2011).

Respecto de la segunda cuestión, relacionada con el alcance y la intencionalidad de la tipología, lo primero que debe entenderse es que esta es de tipo ideal weberiano; es decir, es una simplificación deliberada de una realidad empírica compleja, con el propósito de resaltar ciertos elementos que no son claros en el mundo real (Weber, 2011). Además, la propuesta no pretende abarcar todos los debates de filosofía de la ciencia - ya que no está articulada en esos términos-, sino que busca mostrar un campo de juego nivelado entre posiciones que son susceptibles de comparación y contraste. Sin embargo, esto no significa que cada una de estas posiciones tengan que hacerse conmensurables de forma que permitan algún tipo de prueba empírica sobre ellos.

La formulación de esta tipología fue realizada para rastrear los debates filosóficos importantes para los académicos de las relaciones internacionales. En este trabajo, sin embargo, creemos que también es útil para organizar debates dentro de otros campos disciplinares de las ciencias sociales. En consecuencia, en la siguiente sección mostramos cómo esta tipología puede ser utilizada para ordenar las diversas teorías propuestas en el ámbito de lo que se conoce como análisis de políticas públicas.

\section{LA UTILIDAD DE LA TIPOLOGÍA PARA LAS TEORÍAS DEL ANÁLISIS DE POLÍTICAS}

Arriba mencionamos que el análisis de políticas es un campo relativamente joven, pero esto no significa, en ningún caso, que sea improductivo. A lo largo de los setenta años aproximadamente de desarrollo se ha propuesto un número importante teorías. Estas, como en todos los ámbitos de la ciencia, se pueden dividir en macroteorías, teorías de rango medio y microteorías. En este documento hemos elegido trabajar solo con las macroteorías o, como también se les conoce en la disciplina, marcos analíticos. Es decir, teorías entendidas como ordenación interna de un marco de análisis que permite sistematizar datos observacionales para predecir y explicar eventos empíricos y también para conceptualizar objetos a partir de sus propiedades observables e inobservables (Sayer, 2010).

La elección de las macroteorías está justificada por cuatro razones, dos de carácter científico y dos de naturaleza práctica. La primera de ellas es que los marcos analíticos tienen un mayor nivel de abstracción y jerarquía conceptual para explicar el universo de las políticas públicas (Ostrom, 2007). La segunda es que los marcos, como dispositivos metateóricos, ofrecen la posibilidad a los académicos de discutir cualquier proposición teórica en particular, así como comparar y construir teorías (Bobrow y Dryzek, 1987; Ostrom, 2014). En tercer lugar, los marcos ya han sido objeto de otras sistematizaciones referentes al contexto latinoamericano (Roth, 2014; Fontaine, 2015). Y, finalmente, por la extensión y las características de este trabajo.

Muchos de los libros o manuales de políticas públicas presentan los marcos analíticos como una lista de compra o bote de basura, donde los investigadores pueden 
tomar la teoría que más les convenza (entre otros, Fischer et al., 2007; Goodin et al., 2008; Araral et al., 2012; Cairney, 2020). Uno de los efectos no deseados de este tipo de trabajos es que los investigadores — noveles sobre todo- se pregunten constantemente cuál es la mejor teoría para realizar sus pesquisas sin encontrar respuestas satisfactorias. En ese momento, por lo general, suelen hacer dos cosas: la primera es buscar trabajos o publicaciones temáticamente familiares y adoptar el mismo marco analítico que esas utilizaron, mientras que la segunda es incorporarse a un grupo de trabajo con una línea de investigación, teorías y metodologías establecidas, pero sin tener mayores conocimientos de cómo y para qué se hace lo que se está investigando. Ambas estrategias, aunque funcionales, no son necesariamente las que mejores resultados pueden llegar a producir.

En este escenario es importante señalar que el presente trabajo no tiene por finalidad ofrecer criterios de privilegio para la elección de un marco analítico sobre otro. Por el contrario, se busca mostrar que el campo del análisis de políticas es amplio y que ninguna comprensión filosófica debería dominarlo. Asumiendo ese pluralismo, creemos que son los objetivos del estudio de políticas públicas y el contexto de la investigación los criterios que, a priori, deben guiar la elección del marco apropiado. Asimismo, entendemos que la elección del marco no determina la adopción de un método o métodos específicos, sean cualitativos o cuantitativos, lo que no implica que deban combinarse métodos de diferente naturaleza ontológica dentro de un mismo marco. Contra esa posibilidad sostenemos que, para realizar investigaciones más consistentes, los académicos pueden realizar un ejercicio consciente y transparente de alineación entre estructuras lógicas, teorías y procedimientos de investigación. Para ello, en la tabla 2 proponemos una clasificación de las teorías macro del análisis de política en función de la tipología planteada por Jackson (2016).

TABLA 2.

TiPOLOGÍA APLICADA A LAS TEORÍAS DE ANÁLISIS DE POLÍTICAS

\begin{tabular}{|c|c|c|c|}
\hline & \multicolumn{2}{|c|}{$\begin{array}{l}\text { Relación entre conocimiento y observación } \\
\text { (ontologías científicas) }\end{array}$} \\
\hline & & Fenomenalismo & Transfactualismo \\
\hline \multirow{2}{*}{$\begin{array}{l}\text { Relación entre } \\
\text { el conocedor y } \\
\text { lo conocido } \\
\text { (ontologias } \\
\text { filosóficas) }\end{array}$} & $\begin{array}{c}\text { Dualismo } \\
\text { mente mundo }\end{array}$ & $\begin{array}{l}\frac{\text { Neopositivismo }}{\text { Marco de análisis y desarrollo }} \\
\text { institucional. } \\
\text { Equilibrio puntuado. } \\
\text { Marco de coaliciones promotoras }\end{array}$ & $\begin{array}{l}\frac{\text { Realismo crítico }}{\text { Análisis }} \\
\text { neoinstitucional de } \\
\text { políticas públicas }\end{array}$ \\
\hline & $\begin{array}{c}\text { Monismo } \\
\text { mente mundo }\end{array}$ & $\begin{array}{l}\text { Marco de análisis narrativo de } \\
\text { políticas. } \\
\text { Dependencia de la trayectoria }\end{array}$ & $\begin{array}{l}\text { Reflexivismo } \\
\text { Sociología de la } \\
\text { acción pública. } \\
\text { Análisis deliberativo } \\
\text { de políticas }\end{array}$ \\
\hline
\end{tabular}

Fuente: elaboración propia. 
Antes de explicar la propuesta de tipología aplicada al campo de las políticas públicas son nuevamente necesarias algunas aclaraciones sobre alcance y propósitos: primero, la alineación que aquí se presenta no es una camisa de fuerza ni es de naturaleza mecánica; al contrario, son caminos que pueden conducir a investigaciones congruentes y consistentes. Segundo, y siguiendo el espíritu ideal-típico weberiano, la propuesta de alineación está realizada sobre la mayor cantidad de elementos compartidos o coincidentes. Dicho esto, en las próximas secciones expondremos por qué consideramos que estas teorías se alinean a las metodologías y cuáles son las implicaciones en términos prácticos para la investigación. En primer lugar se presentarán los marcos explicativos (neopositivistas y realistas) y luego los marcos interpretativos (analiticistas y reflexivistas).

\section{Teorias neopositivistas}

El término neopositivismo suele asociarse rápidamente al uso extensivo de modelización econométrica, semiexperimentos y análisis coste-beneficio (Fontaine, 2020); en definitiva, lo que se considera corriente dominante (mainstream) dentro del campo de las políticas públicas. Sin embargo, más allá de este tipo de investigaciones y métodos derivados de la escuela de la elección pública (Blankart y Koester, 2006), se encuentran un conjunto de teorías para analizar las políticas que fueron formuladas siguiendo argumentos metodológicos neopositivistas. Estas teorías son, como mencionamos en la tabla 2, el marco de análisis y desarrollo institucional (Ostrom, 2014), el equilibrio puntuado (Jones y Baumgartner, 2005) y el marco de coaliciones promotoras (Sabatier, 2010).

El primer elemento identificable de las teorías neopositivistas son las conjeturas no falseadas como estado del conocimiento. Esto significa que lo que consideran conocimiento válido para desarrollar o hacer avanzar la ciencia (Popper, 2008) es una afirmación que aún no ha sido refutada. El procedimiento privilegiado para producir y evaluar esas afirmaciones es el testeo de hipótesis. Ahora bien, cuando se piensa en testeo de hipótesis, lo primero que viene a la mente es la comparación de grandes casos a través de modelos econométricos. Sin embargo, de estas teorías, solo el equilibrio puntuado (EP) privilegia este tipo de procedimientos. Aunque es necesario aclarar que toma distancia de la modelización de regresiones para describir tendencias o covariaciones, y se enfoca en la predicción de momentos (puntos) para explicar las decisiones (Fontaine, 2015).

Por otra parte, tanto el marco de análisis y desarrollo institucional (ADI) como el marco de coaliciones promotoras (MCP) se han desarrollado a través del uso de métodos cualitativos y cuantitativos centrados en casos, para producir y testear las hipótesis. El ADI nace de la revisión de más de quinientos casos estudiados por distintos grupos de investigación, con el objetivo de identificar las variables claves que permiten la organización de individuos o aquellas que las limitan. Para ello, el ADI trata de «hacer posible el análisis de sistemas integrados a partir de un clúster de 
variables, cada una de las cuales puede desglosarse, dependiendo de la pregunta de interés» (Ostrom, 2014: 23). En el núcleo del marco está la «situación de acción», es decir, el espacio social donde los actores interactúan, se resuelve el problema de los bienes comunes y se intercambian bienes y servicios. Además, el marco contempla un conjunto de variables externas que afectan a la situación de acción y son identificadas como: condiciones biofísicas, atributos de la comunidad y reglas de uso. Luego de elaborado el marco, este se aplicó a múltiples escenarios empíricos ${ }^{2}$ con el fin de verificar y ajustar las hipótesis propuestas.

El MCP, por otra parte, nace como parte de la insatisfacción de Paul Sabatier (1988) con el «ciclo de las políticas» y de su interés por resolver problemas «insolubles». Tiene tres niveles (macro, meso y micro) que son sus piedras angulares. En el nivel macro está el subsistema de políticas que es afectado por factores externos del sistema político y socioeconómico. El nivel micro es un «modelo del individuo» tomado de la psicología social. El nivel meso son las coaliciones promotoras donde se juntan la multiplicidad de actores del subsistema (Sabatier y Weible, 2010). Los desarrollos del MCP a lo largo de la década de 1990, llevaron a determinar doce hipótesis - y sus reformulaciones - sobre el comportamiento del subsistema de políticas (Rubio y Rosero, 2010). Con ello, se puede observar que la lógica del MCP y ADI es similar, y consiste en que los investigadores utilicen los marcos para comprobar o falsear en sus casos de estudio el cumplimiento de alguna o algunas de las hipótesis formuladas.

De esta forma es posible afirmar que lo que permite identificar a una teoría neopositivista no es el método cuantitativo o cualitativo que privilegia, sino la pretensión de generalización empírica como forma de entender la causalidad y la subsunción en leyes generales como procedimiento para la explicación causal. En términos prácticos para la investigación esto significa que los investigadores deberían comprobar el cumplimiento o no de las hipótesis (leyes universales) en sus casos y difundir los resultados, de manera que estos sean útiles para alimentar una agenda de investigación más amplia.

El último elemento compartido de las teorías neopositivistas es la denominación de las unidades de análisis de su objeto de estudio: las variables ${ }^{3}$. En el caso del MCP, por ejemplo, el subsistema de políticas es tratado como variable dependiente y lo que se pretende lograr con la investigación es demostrar que las variables independientes explican el subsistema (Sabatier, 2010). Esas variables independientes pueden ser

2. Si bien el marco comenzó a utilizarse para estudiar los servicios policiales en áreas metropolitanas, las aplicaciones más conocidas son las relacionadas con los arreglos institucionales sobre los recursos de uso común. En la actualidad, su uso se ha extendido a una impresionante diversidad de temáticas.

3. Las variables son comprendidas desde una perspectiva neopositivista como un término que implica la posibilidad de descomponer un fenómeno a través del reconocimiento de los atributos específicos que conforman los objetos de estudio, lo que conlleva a que exista una lógica particular de proceder en las investigaciones (King et al., 2000: 56-57). 
endógenas (transformaciones del subsistema o de sus coaliciones) o exógenas (condiciones socioeconómicas, coaliciones del sistema de gobierno o decisiones e impactos de otros subsistemas). En el ADI, Ostrom (2014) se pregunta si es posible encontrar unidades universales (variables) que estructuren las interacciones humanas, cualesquiera que estas sean. Esas interacciones humanas son identificadas como una situación de acción (variable dependiente) que puede ser impactada por las reglas, los atributos de la comunidad y los atributos biofísicos y los materiales del contexto o entorno (tres tipos de variables independientes). Finalmente, el EP busca explicar y predecir cambios radicales (puntos) a través de variables endógenas, como el ciclo de atención del problema, y exógenas, como la organización del Estado (Fontaine, 2015: 159). De esta manera, plantean la hipótesis de que las instituciones se ajustan constantemente a los contextos cambiantes, por lo que la configuración de una agenda política tiende a ser un proceso errático que sigue una distribución accidentada (Jones y Baumgartner, 2005).

Lo anterior evidencia que no es suficiente la distinción entre los términos cuantitativo y cualitativo para diferenciar teorías neopositivistas de, por ejemplo, constructivistas. La metodología neopositivista no se limita a comparaciones de grandes casos a través de estadísticas; también contempla estudios de casos individuales. Sin embargo, lo último solo es posible si los casos están explícitamente relacionados con generalizaciones (casos cruciales), funcionando como inputs para la evaluación de propiedades de resultados previos; o como componente de una estrategia comparativa amplia (Jackson, 2016: 221).

\section{Teorias realistas}

El segundo tipo de marcos explicativos de la tipología son los realistas. El realismo es una posición filosófica consolidada e identificable; sin embargo, no goza de suficiente visibilidad e individualización dentro del análisis de políticas. Esto puede deberse a dos razones: la primera es que las críticas que se realizan desde los marcos o teorías interpretativas (analíticas o reflexivas) suelen agrupar a todas las teorías explicativas en la categoría neopositivista, mientras que la segunda se relaciona con la propia naturaleza de la comprensión de los objetos de investigación y el poco conocimiento que hasta ahora se tiene sobre los procedimientos de trabajo.

El realismo surgió como crítica al positivismo que, según Bhaskar, había «usurpado el título de ciencia» (2008: 29). Es fácil suponer que entre ambas posiciones existen diferencias sustanciales en la forma de comprender la ciencia y sus métodos. La principal es sobre los objetos del conocimiento. Los realistas argumentaron que la idea de ver los objetos únicamente como hechos que hablan por sí mismos y que pueden ser colectados como datos era una posición ingenua. En cambio, explicaron que debe realizarse una distinción entre dos tipos: a) objetos de pensamiento basados en teoría y conocimiento factual, observacional y empírico, y b) objetos reales basados en los hechos como cosas, propiedades o estados del mundo (Sayer, 2010). 
La distinción entre los objetos se traduce en lógicas totalmente diferentes de producción del conocimiento. En consecuencia, el estado del conocimiento de la metodología realista no es una conjetura no falseada (como en el neopositivismo), sino un mejor acercamiento al mundo. Lograr aproximarse de mejor manera al mundo implica entender que este no se reduce a sus estructuras materiales, sino que deben descubrirse las propiedades disposicionales profundas de los objetos (Jackson, 2016). Esto significa que la producción de conocimiento involucra conceptos y fenómenos interdependientes y no solamente eventos discretos. A manera de ejemplo, donde los positivistas explican la existencia de una correlación entre una variable $\mathrm{X}$ (llamémosla Voice and accountability) y una variable Y (llamémosla Control of Corruption) mediante el análisis de una base de datos, a los realistas les interesa más el proceso (donde se incluyen entidades, instituciones, actores, fuerzas causales) que permite afirmar que $\mathrm{X}$ es una causa genuina de $\mathrm{Y}$ en un caso o conjunto reducido de casos. Nuevamente, esto no debe confundirse con una división simplista de métodos cuantitativos y cualitativos. Al igual que sucede con las teorías neopositivistas, los realistas pueden utilizar ambos tipos de métodos para lograr sus propósitos de investigación (Fontaine et al., 2018).

La teoría que consideramos que se alinea con esta metodología es el análisis neoinstitucional de políticas públicas (ANP). El ANP, en este trabajo, lo entendemos a partir del trabajo seminal de March y Olsen (1984). Esta aclaración es necesaria debido a que existen múltiples aproximaciones del neoinstitucionalismo (Hall y Taylor, 1996; Peters, 2003) y no todas son compatibles con la metodología realista. En ese sentido, es notable que las teorías neopositivistas tienen características compartidas con el neoinstitucionalismo de elección racional, mientras que las teorías analíticas comparten características con el neoinstitucionalismo histórico. El ANP, como aquí lo comprendemos, no pretende explicar los resultados de las instituciones creadas por los actores para solucionar un problema (elección racional), ni interpretar la trayectoria histórica que provocó determinado resultado (histórico). El objetivo del ANP es explicar cómo se institucionalizan las políticas públicas a través de procesos causales concretos.

El elemento más obvio de la alineación del ANP con el realismo es el estudio de las instituciones como objetos del conocimiento que no son directamente observables, pero si detectables. Las instituciones, desde esta teoría, son conceptualizadas como una colección relativamente duradera de «reglas y prácticas organizadas, incrustadas en estructuras de significado y recursos, que son relativamente invariantes frente a la rotación de individuos y relativamente resistentes a las preferencias y expectativas idiosincrásicas de los actores y las circunstancias externas cambiantes» (March y Olsen, 2009: 1). Esta comprensión de las instituciones es diferente a la del resto de los científicos políticos y, también, más amplia. En ella se incluyen sistemas de símbolos, guías cognitivas y plantillas morales encargadas de proporcionar las «estructuras de significado» que moldean la acción humana (Hall y Taylor, 1996).

Las instituciones, en consecuencia, son el concepto que ayuda a desarrollar una correcta comprensión de los fenómenos y también, en cierto sentido, anticipar lo que puede o no suceder (Offe, 1996). Esta idea está sujeta a una «lógica de lo adecuado» 
que determina las acciones apropiadas entre roles e instituciones (March y Olsen, 1989). Es decir, los elementos de orden y previsibilidad se crean mediante la definición de un conjunto de expectativas de comportamiento que se consideran correctas (Peters, 2003; Lowndes, 2019). Las expectativas adecuadas, a su vez, se internalizan mediante procesos de socialización (Olsen, 2008) y estructuran el comportamiento, las creencias y la acción organizada dentro de los sistemas políticos, formando, habilitando y restringiendo a los actores (Scott, 2014; Gruber et al., 2015).

El procedimiento para evaluar las afirmaciones de las investigaciones que utilizan esta teoría es a través de ambientes controlados como los laboratorios de políticas (Olejniczak et al., 2019) o por medio de argumentos trascendentales ${ }^{4}$ (Cabrera, 2007). El primer tipo de procedimiento es prácticamente inexistente dentro del contexto latinoamericano, ya que requiere de ingentes recursos y colaboración entre Gobiernos, actores no estatales, organizaciones internacionales y comunidades locales, lo cual suele ser muy difícil de lograr. El segundo tipo de procedimiento, en cambio, es frecuentemente utilizado en escuelas de posgrado de la región para la elaboración de trabajos de graduación o desarrollo de agendas de investigación (Muñoz, 2011; Zabala-Peñafiel, 2017; Rodrigues, 2018; Fontaine, 2019).

Las investigaciones que utilizan el ANP rechazan la generalización empírica y ponen el foco en ofrecer explicaciones causales de tipo disposicional ${ }^{5}$. Estas disposiciones son propiedades que forman el núcleo esencial del objeto, y la «realidad» del mismo está determinada por las condiciones bajo las cuales esas propiedades producen efectos (Mumford, 1998; Guirado, 2014). En términos prácticos, esto significa que el procedimiento para determinar la causalidad implica identificar el conjunto de condiciones INUS - parte insuficiente pero necesaria de una condición que es innecesaria pero suficiente (Mackie, 1965: 245) — que explican el resultado de la política. Las investigaciones que utilizan el ANP no solo pretenden explicar la política pública como resultado de un diseño institucional determinado, sino que se interesan también por los efectos de las políticas en la sociedad (Eslava, 2010). La dirección de la causalidad en uno u otro sentido depende de los intereses del investigador y de su pregunta de investigación.

Determinar propiedades disposicionales requiere de métodos diferentes a la modelización de regresiones. El objetivo no es comprobar covariación entre $\mathrm{X}$ e $\mathrm{Y}$, sino explicar por qué X produce $\mathrm{Y}$. Uno de los métodos para lograr este objetivo es el rastreo de procesos (process tracing) de tipo prueba de teoría ${ }^{6}$. Este método es útil para develar los mecanismos causales que operaron dentro de un caso o un conjunto de casos deter-

4. Tipo de argumentación filosófica que pretende establecer las condiciones necesarias de la experiencia.

5. Una disposición se entiende, de manera simple, como «la capacidad de un sistema de actuar sobre otro modificándolo» (Álvarez, 2018: 30).

6. Según los autores, el process tracing no es un método único, como suele ser presentado. Para ellos existen tres variantes: prueba de teoría, construcción de teoría y explicación de resultado. La utilización de una u otra variante depende de la pregunta del investigador, así como de los objetivos teóricos y metodológicos de su trabajo. 
minados. La comprensión mecánica de la causalidad permite observar el proceso mediante el cual las fuerzas causales se transmiten a través de una serie de partes interconectadas en un mecanismo, llegando a producir un resultado determinado (Beach y Pedersen, 2019). Es decir, el funcionamiento de un grupo de elementos (INUS) que producen un efecto que no es inherente a ninguno de ellos en singular. Para la comprobación formal de la presencia de estos mecanismos pueden utilizarse procedimientos cualitativos como entrevistas o análisis documental y también cuantitativos como la lógica bayesiana (Bennet y Checkel, 2015). En definitiva, los mecanismos causales son simplificaciones de la realidad — no observables, pero sí detectables - que permiten abrir la caja negra de la causalidad tanto como sea posible y detectar fuerzas causales que explican la ocurrencia de un fenómeno determinado. En este sentido, la naturaleza ontológica del método es compatible y se alinea con la metodología realista.

Un elemento particular de las investigaciones del ANP es la comprensión de las políticas públicas como sistemas abiertos ${ }^{7}$. Un sistema abierto es aquel donde no se cumple la condición intrínseca o la extrínseca de cierre del sistema. Esto significa que no debe haber variación cualitativa en el objeto poseedor de los poderes causales (intrínseca) y que la relación entre el mecanismo causal y las condiciones externas que influyen en la operación de este deben ser constantes (extrínseca). En el caso de que ambas condiciones de cierre se cumplan, se está en presencia de un sistema cerrado que produce regularidades. Sin embargo, desde el ANP se entiende que la política pública es un sistema que generalmente viola la condición extrínseca, por lo que las regularidades que pudieran producirse son solo «aproximadas y fugaces» (Sayer, 2010: 122). Debido a esta naturaleza contexto-dependiente de la forma en que las propiedades disposicionales se manifiestan en el mundo, las investigaciones no conducen a leyes empíricas generales.

Finalmente, cabe señalar que la utilización de métodos para develar mecanismos causales dentro de los casos o la imposibilidad de aportar explicaciones tipo-ley no impide realizar comparaciones; al contrario, cada vez es mayor el esfuerzo por multiplicar los estudios comparativos (Peters y Fontaine, 2020). El tipo de comparación que se realiza es por contraste y con el propósito de elucidar poderes causales (Jackson, 2016). Con este se pretende delinear las capacidades de las propiedades disposicionales para explicar de qué manera los diferentes diseños institucionales facilitan o restringen determinados resultados.

\section{Teorias analiticistas}

Los enfoques monistas en filosofía de la ciencia suelen agruparse, con bastante frecuencia, bajo el término constructivismo. Dentro de esta corriente, sin embargo, se encuentran posiciones con ontologías científicas diferentes, tanto fenomenológicas

7. Si bien el ADI y el MCP entienden a las políticas públicas como sistemas abiertos, estos proponen un conjunto de variables que permiten controlar la influencia de los factores externos (contexto), o cerrar el sistema y, de esta manera, producir explicaciones generalizables. 
como transfactuales. Con esta preocupación en mente, Jackson (2016) propone una división de dos tipos para los enfoques monistas. El primero de ellos es el analiticismo, el cual combina el monismo mente-mundo con el fenomenalismo y cuya denominación está inspirada en la filosofía analítica y en la división de sistemas (concretos y analíticos) formulada por Parsons (1961).

La postura analítica, al igual que el neopositivismo, tiene interés por objetos de investigación que son directamente observables. Se diferencian en que para el analiticismo es la experiencia del investigador lo que permite situar la importancia y el significado de los objetos. De esta manera, el primer elemento que posibilita identificar las teorías que se alinean con esta metodología son las constataciones útiles como estado del conocimiento. Con esto queremos decir que el conocimiento válido para estas teorías es una organización sistemática y coherente del material empírico de casos específicos.

El procedimiento para la producción de ese conocimiento es a través de narrativas analíticas. Esas narrativas suelen construirse a través del uso de herramientas formales que reducen la posibilidad de cometer errores lógicos. Por ejemplo, uno de los métodos privilegiados es el rastreo de procesos (process tracing), pero en este caso, de tipo explicación de resultados (Beach y Pedersen, 2019) ${ }^{8}$. Con este procedimiento metodológico los investigadores intentan rastrear el complejo conglomerado de eventos que, de manera secuencial y concatenada, produjeron un resultado en específico. Esto hace que las investigaciones se asemejen a descripciones de tipo historiográfico. Las narrativas producidas, como se verá más adelante, suelen tener ambiciones teóricas que van más allá del caso único.

Dentro del conjunto de teorías de las políticas públicas, una de las que se alinea con esta posición filosófica es el análisis narrativo de políticas (ANAR) formulado por Emery Roe (1994). Según esta teoría, el trabajo de un analista se realiza mediante un estudio a profundidad de un caso que comienza con la identificación de las narrativas dominantes sobre un problema de política. Posteriormente a ello debe identificar narrativas no dominantes o aquellas que van en contra de las anteriores (las no-historias o contrahistorias). Finalmente, debe comparar los dos tipos de narrativas y generar una metanarrativa que contribuya a reformular el problema, de manera que sea más dócil de resolver.

En términos de implicaciones prácticas, el primer paso del investigador que utiliza el ANAR es realizar entrevistas. Mediante esta técnica, los actores facilitan al analista un conjunto de elementos que permiten reconstruir la historia desde dos puntos de

8. La variante prueba de teoría mencionada para el realismo es muy diferente a la de explicación de resultados. En la prueba de teoría el seguimiento del proceso es objetivista; es decir, su utilización implica una separación entre el investigador y el objeto investigado. Además, el proceso se entiende de forma diacrónica. Mientras que el uso de la variante explicación de resultados implica subjetividad del investigador en cuanto realiza un ejercicio de interpretación de hechos históricos de manera sincrónica y mediante ese ejercicio le atribuye conexiones causales a esos eventos. 
vista diferentes. Una vez que el investigador ha identificado los dos tipos de configuraciones relacionadas con el problema, realiza un análisis de redes que le permita identificar las interrelaciones entre las narrativas, lo cual debe dar lugar a comprender el eje de la controversia. Por último, establece cuáles son los principales elementos sobre los que existe incertidumbre y emite una serie de recomendaciones según su experiencia. Esta explicación puede generar la ilusión de que el elemento central de las investigaciones es generar soluciones que contribuyan a la toma de decisiones. Si bien esa fue una de las intenciones de Roe en la formulación original, la aplicación ha tendido más a la construcción de historias sobre cómo se llegó a un determinado estado de cosas, y bastante menos a proponer soluciones específicas a los problemas de las políticas públicas.

El segundo elemento identificable de las teorías analíticas es la comprensión de la causalidad mediante tipos ideales. Comprender la causalidad de esta forma implica construir un tipo ideal o varios tipos ideales en combinación que arrojen luz sobre lo que sucedió en un caso en específico. En el ejemplo, las narrativas y las contranarrativas son determinadas por el investigador como tipos ideales en función a su experiencia y su subjetividad. Debe recordarse aquí a Weber ([1949] 2011) para pensar en los tipos ideales como construcciones analíticas que no se encuentran en la realidad empírica; o lo que es lo mismo, como una simplificación deliberada de situaciones reales y complejas que tienen el objetivo académico de llamar la atención sobre determinados aspectos en perjuicio de otros. Una ilustración del uso de tipos ideales es el conocido trabajo de Esping-Andersen (1993) sobre los tipos de Estados que existen en el mundo capitalista (occidental) y sus características. Con similares objetivos, Barba (2019) ha determinado tipos de regímenes de bienestar en América Latina. Y, como ejemplo distante del ámbito de las políticas sociales, Peters y Pierre (2020) clasifican los tipos de regímenes populistas y argumentan las implicaciones de cada uno en la Administración y políticas públicas.

El tercer elemento identificable de estas teorías es el procedimiento para la explicación causal, ya que este suele realizarse a través de análisis de contrafácticos?. Esto significa que la individualización de las causas que se utilizan en la narrativa surge de un proceso de reflexión mediante el cual el investigador se pregunta si el resultado hubiese sido posible ante la ausencia de esos factores, o qué hubiera sucedido en presencia de otros factores que en el caso que analiza estuvieron ausentes. Intentaremos aclarar este procedimiento a través de la teoría de la dependencia de la trayectoria $(\mathrm{DDT})^{10}$.

La DDT es una teoría que surge dentro del campo de la sociología histórica (Fontaine, 2015) como una aportación para el análisis de procesos políticos. La idea fundamental es ayudar a comprender cómo determinados eventos que sucedieron en

9. De manera diferente a como es utilizado por teorías neopositivistas.

10. Una explicación más amplia de la DDT como marco analítico se puede encontrar en el texto de Fontaine (2015). 
un tiempo pasado tienen influencia en resultados futuros e históricamente distantes (Mahoney, 2000). Para ello, las investigaciones deben identificar los momentos históricos iniciales de una secuencia; los que se conocen como coyunturas críticas ${ }^{11}$. Estas coyunturas críticas son eventos contingentes que no pueden o deben ser explicados por eventos previos, sobre todo para que el análisis no sea un recuento ad infinitum. Por último, la teoría plantea que las secuencias están marcadas por una «inercia», es decir, que una vez que los procesos echan a andar tienden a mantener un movimiento que persigue un resultado particular (Mahoney y Villegas, 2007).

Las coyunturas críticas suelen ser determinadas por los investigadores mediante análisis contrafáctico. El procedimiento es útil para comprender cómo se llegó a un resultado específico partiendo desde A y también para imaginar a qué otro resultado se podía haber llegado en caso de haber partido desde una opción B. Es importante entender que la determinación de las coyunturas críticas es producto de inferencias parcializadas, que el investigador realiza y que pueden ser contestadas por otros investigadores. En ese sentido, no son verdaderas ni falsas. Por ello creemos que es un error que las investigaciones que utilizan esta teoría se planteen comprobar hipótesis de investigación; sobre todo porque al partir de una ontología filosófica monista, la idea de un mundo fuera de la mente carece de sentido.

El conjunto de ideas hasta aquí expuestas indica que las investigaciones que siguen la metodología analiticista son fundamentalmente estudios de caso. Si bien es cierto que muchas de ellas hacen énfasis en este tipo de estudios, también la comparación es posible. No obstante, el propósito de la comparación dista mucho del testeo de covariaciones hipotéticas del neopositivismo. En cambio, esta se realiza mediante procesos de individualización donde se resaltan características concretas de los casos con el propósito de especificar configuraciones particulares (Jackson, 2016), o se especifican las configuraciones particulares y luego se individualizan los casos (Beach y Pedersen, 2019). En definitiva, son operaciones de inferencia abductiva.

\section{Teorías reflexivas}

La reflexividad es la metodología que surge de la combinación entre el monismo mente-mundo y una noción transfactual del conocimiento. Los investigadores que se alinean con la reflexividad entienden que el conocimiento es inseparable de la posición social que ocupan y las prácticas organizacionales en las que participa. Es decir, para ellos conocer y cambiar el mundo son dos actividades inseparables. Sin embargo, esta es una posición que entraña tensión debido a que si el conocimiento es producto de la

11. Una coyuntura crítica es la combinación de dos o más factores que caracterizan a una situación en un momento determinado. Implican la adopción de un arreglo particular entre dos o más alternativas (de ahí la denominación crítica) que puede tener un fuerte impacto en resultados posteriores. 
experiencia — característica del monismo—-, no está muy claro qué significa que la trascienda (Jackson, 2016).

El conocimiento que se considera válido (estado del conocimiento) adopta la forma de dispositivos que incrementan la autoconciencia; es decir, debe mejorar la capacidad de reflexionar de los investigadores. Lograr ese conocimiento implica un examen detallado y teorización sobre la base de condiciones sociales e históricas de posibilidad. El proceso es dialéctico. La interpretación de las condiciones contribuye a transformar las propias condiciones, creando un nuevo estado, una «síntesis» en términos hegelianos.

Dentro del campo del análisis de políticas una de las teorías reflexivas es la sociología de la acción pública (SAP). Para esta teoría las políticas públicas no son una variable dependiente o independiente, sino que son el producto de la acción colectiva que participa en la creación de un determinado orden social y político (Lascoumes y Le Gàles, 2012). De esta manera, se entiende que otorgan dirección a la sociedad y regulan las tensiones o conflictos entre los grupos que la conforman. Sin embargo, las políticas no se comprenden como un objeto distante que produce el Gobierno de manera top down, sino que son procesos donde actores públicos y privados desarrollan estrategias conjuntas desde abajo. Estos procesos fluyen entre confrontaciones, negociaciones y acuerdos entre los actores (Fontaine, 2015). Entonces, el sentido de la investigación está dirigido a la construcción de «marcos de interpretación del mundo» (Muller, 2006: 98) que medien los conflictos y, de esta forma, hacer posibles las políticas.

El análisis deliberativo de políticas (ADP) es también afín a la reflexividad. Esta teoría plantea que «la política está hecha de palabras» (Majone, 1997: 35), por lo que analizarla es sinónimo de interpretar los discursos. Este es un ejercicio complejo debido a que las políticas públicas son problemas transcientificos. Es decir, problemas que no son ni puramente técnicos ni puramente políticos. Al tener esta característica, las políticas son un espacio donde confluyen discursos - o marcos de sentido- científicos, técnicos y políticos, lo que las hace propensas al conflicto. En este sentido, el rol investigador es comprender todos los discursos en disputa y producir evidencias y argumentos que ayuden a la deliberación en el debate público (Fischer, 2003).

Otro de los elementos que permiten identificar las investigaciones reflexivas es la comprensión dialéctica de la causalidad. Como anteriormente referimos, el lugar de la investigación está relacionado dialécticamente con sus condiciones sociales de producción a través de un proceso de identificación (o interpretación) que potencialmente contribuye a su transformación. De esta manera, las investigaciones no son solo descriptivas, sino que son articulaciones realizadas desde el presente y están dirigidas desde el pasado hacia el futuro (Jackson, 2016). El procedimiento para la explicación causal consiste en develar tensiones no resueltas. En el caso de la SAP, el procedimiento se materializa a través del modelo referencial (Muller, 2006). Este modelo está compuesto por tres elementos: referencial global, referencial sectorial y la relación entre ellos. El referencial global son las visiones generales, imágenes cognitivas o creencias básicas que organizan las percepciones sociales sobre el sistema. Mientras que el 
referencial sectorial corresponde a las normas, valores o imágenes de un sector específico de las políticas, una disciplina o profesión. La relación entre ambos referenciales suele entrañar conflictos ${ }^{12}$ y el rol de la investigación es desvelarlos y, con ello, tratar de mediar. Las investigaciones que siguen el ADP proceden de manera bastante similar. Desde esta teoría se parte de entender que el análisis de política tiene una función fundamentalmente argumentativa. El ejercicio de la argumentación desde ahí tiende más a la categorización de problemas que a su solución (Fontaine, 2015). Es decir, el análisis tiene el objetivo de delimitar los problemas e identificar sus causas a través de la interpretación del mundo social y de los discursos de los actores en disputa.

De esta manera, ambas teorías coinciden en que el producto de la investigación se relaciona con la práctica de las políticas a través de su incidencia en procesos de deliberación, proposición y persuasión entre actores estatales y no estatales. También concuerdan en que la producción de un conocimiento útil para ese fin depende de la relación de los investigadores con el conocimiento práctico cotidiano y con los grupos sociales que lo poseen, lo que implica identificación, apropiación y sentido de pertenencia con el mundo que investigan. Esto hace, por otra parte, que los investigadores estén expuestos siempre a la crítica respecto a que sus trabajos son intervenciones políticas de tipo partidista o que están motivados por sentido común y no por criterios científicos.

$\mathrm{Al}$ igual que sucede con la investigación alineada con el analiticismo, la mayoría de los trabajos que siguen enfoques reflexivos tienden a realizar estudios de caso (Echeverría y Maturana, 2015; Ortega-Bolaños y Alba-Muñoz, 2017), aunque las comparaciones también son posibles (Buitrago, 2015; Fair, 2019). Sin embargo, las comparaciones aquí se realizan con el objetivo bien de desnaturalizar un discurso referencial hegemónico en algún sector de las políticas, o bien de incorporar elementos a la deliberación que no estaban siendo tomados en cuenta con la intención de provocar el cambio social.

\section{CONCLUSIONES}

Dentro del análisis de políticas se han propuesto diversos marcos analíticos para sistematizar observaciones, predecir y explicar eventos empíricos y conceptualizar objetos a partir de sus propiedades observables e inobservables. Estos marcos son útiles también para comparar y construir teorías; sin embargo, aún persisten vacíos respecto a cómo lograr alinear las estructuras ontológicas, metodológicas y teóricas en la investigación. La

12. Un ejemplo de conflicto entre ambos referenciales se puede observar en el intento de rediseño de la política energética de Ecuador en el mes de octubre de 2019; específicamente respecto al instrumento subsidios de combustibles Extra, EcoPaís y Diesel. El referencial sectorial visibilizó y argumentó que el subsidio es una distorsión de la economía nacional, mientras que el referencial global argumentaba que era un instrumento redistributivo y que su eliminación era un acto de violencia económica contra los sectores más pobres de la sociedad. El conflicto en esa ocasión fue violento y, aún hoy, no resuelto. 
tipología propuesta en este trabajo tiene como principal objetivo contribuir a completar esos vacíos. Para lograrlo, la clasificación ordenó el estado del conocimiento de las principales macroteorías de las políticas a través de las visiones de mundo y de la ciencia que subyacen a dichas reflexiones teóricas. La finalidad de esa organización no es hacer competir, comparar o jerarquizar teorías, sino que se busca dar cuenta de la pluralidad del campo y de la justificación interna de las propuestas.

La clasificación permite aclarar las discusiones en el campo, los objetivos de los estudios y los métodos que utilizan. Esto facilita, en primer lugar, comprender las implicaciones prácticas que enfrentan las investigaciones y justificar de mejor manera por qué se toman dichas decisiones. Asimismo, con este aporte pretendemos delinear caminos que conduzcan a investigaciones que logren desarrollar mayores niveles de consistencia, coherencia y congruencia entre la comprensión del objeto, el procedimiento y el resultado. Finalmente, tratamos de mostrar que el desarrollo del campo del análisis de política no debe depender de críticas que se realicen entre investigaciones que partan de cuestionamientos sobre los supuestos ontológicos y metodológicos, sino que requiere de ejercicios que permitan hacer comprensibles las afirmaciones entre los distintos lenguajes que coexisten.

\section{Referencias}

Aguilar, Luis. 2007. «El aporte de la Política Pública y de la Nueva Gestión Pública a la gobernanza", Revista del CLAD Reforma y Democracia, 39: 5-32.

Álvarez Toledo, Sebastián. 2018. Disposiciones y puntos de vista causales. Daimon Revista Internacional de Filosofia, 75: 27-41. Disponible en: https://doi.org/ 10.6018/daimon/332161.

Araral, Eduardo, Scott Fritzen, Michael Howlett, M. Ramesh y Xun Wu. 2012. Routledge Handbook of Public Policy. Abingdon: Routledge. Disponible en: https://doi. org/10.4324/9780203097571.

Barba, Carlos. 2019. "Welfare regimes in Latin America: Thirty years of social reforms and conflicting paradigms», en Gibrán Cruz-Martínez (ed.), Welfare and Social Protection in Contemporary Latin America. Nueva York: Routledge.

Beach, Derek y Rasmus Brun Pedersen. 2019. Process-Tracing Methods. Foundations and Guidelines. Michigan: University Michigan Press. Disponible en: https://doi. org/10.3998/mpub.10072208.

Bennett, Andrew y Jeffrey Checkel. 2015. Process Tracing: From Metaphor to Analytic Tool. Cambridge: Cambridge University Press. Disponible en: https://doi. org/10.1017/CBO9781139858472.

Bhaskar, Roy. 2008. A Realist Theory of Science. Nueva York: Routledge.

Blankart, Charles B. y Gerrit B. Koester. 2006. «Political Economics versus Public Choice Two views of political economy in competition", KYKLOS, International Review for Social Sciences, 59 (2): 171-200. Disponible en: https://doi.org/10.1111/ j.1467-6435.2006.00330.x. 
Bobrow, Davis B. y John S. Dryzek. 1987. Policy Analysis by Design. Pittsburg: University of Pittsburg Press.

Buitrago, Yudi M. 2015. "Ideas, discurso y proceso de reforma en el sector salud. Colombia y Ecuador en perspectiva comparada", Mundos Plurales, Revista Latinoamericana de Politicas y Acción Pública, 2 (2): 51-69. Disponible en: https://doi. org/10.17141/mundosplurales.2.2015.1984.

Cabrera, Isabel. 2007. "Argumentos trascendentales. O cómo no perderse en el laberinto de las modalidades», en Isabel Cabrera (comp.), Argumentos trascendentales. México DF: Instituto de Investigaciones Filosóficas.

Cairney, Paul. 2020. Understanding public policy. Theories and Issues (2a ed.). Londres: Red Globe Press.

Echeverría, Genoveva y José M. Maturana. 2015. "Análisis crítico del discurso de políticas públicas en diversidad sexual en Chile», Universitas Psychologica, 14 (4): 1485-1498. Disponible en: http://dx.doi.org/10.11144/Javeriana.up14-4.acdp.

Eslava, Adolfo. 2010. "Análisis neoinstitucional de políticas», en Andre-Noël Roth (ed.), Enfoques para el análisis de politicas. Bogotá: Universidad Nacional de Colombia.

Esping-Andersen, Gøsta. 1993. Los tres mundos del Estado de bienestar. Valencia: Edicions Alfons el Magnànim.

Fair, Hernán. 2019. «Análisis Político del Discurso e investigación empírica: herramientas teóricas y estrategias metodológicas para estudiar identidades y procesos políticos desde América Latina», Ciencia Política, 14 (27): 47-90. Disponible en: https://doi.org/10.15446/cp.v14n27.73323.

Fischer, Frank. 2003. Reframing Public Policy Discursive Politics and Deliberative Practices. Oxford: Oxford University Press.

Fischer, Frank, Gerald J. Miller y Mara S. Sidney. 2007. Handbook of Public Policy Analysis Theory, Politics, and Methods. Nueva York: Routledge. Disponible en: https://doi.org/10.1201/9781420017007.

Fontaine, Guillaume. 2015. El Análisis de Politicas Públicas: Conceptos, Teorías y Métodos. Barcelona: Anthropos-FLACSO Ecuador.

Fontaine, Guillaume. 2019. "Una aproximación realista al diseño de políticas públicas», Cuadernos del CENDES, 36 (102): 117-148.

Fontaine, Guillaume. 2020. "The contribution of policy design to realist evaluation», Evaluation, 26 (3): 296-314. Disponible en: https://doi.org/10.1177/135638902 0902496.

Fontaine, Guillaume, Iván Narváez y Bayron Paz. 2018. «Explaining public accountability deficit in extractive policies: The Ecuadorian case», The Extractive Industries and Society, 5 (1): 190-200. Disponible en: https://doi.org/10.1016/j. exis.2017.11.005.

Goodin, Robert E., Michael Moran y Martin Rein. 2008. The Oxford Handbook of Public Policy. Oxford: Oxford University Press. Disponible en: https://doi. org/10.1093/oxfordhb/9780199548453.001.0001. 
Gruber, Oliver, Sieglinde Rosenberger y Astrid Mattes. 2015. «How institutional actors matter. The impact of governmental innovation on immigrant integration policies in Austria», en 9th ECPR General Conference. Panel P14, Explaining Public Policy Change I. Montreal: Université de Montréal.

Guirado, Matías. 2014. "Metafísica disposicional y monismo neutral», Mutatis Mutandis: Revista Internacional de Filosofía, 1 (3): 11-27.

Hall, Peter y Rosemary Taylor. 1996. «Political Science and the Three New Institutionalisms», Political Studies, 44 (5): 936-957. Disponible en: https://doi. org/10.1111/j.1467-9248.1996.tb00343.x.

Jackson, Patrick. T. 2016. The conduct of inquiry in international relations: Philosophy of science and its implications for the study of world politics (2nd edition). Londres: Routledge.

Jones, Bryan D. y Frank R. Baumgartner, 2005. The Politics of Attention: How Government Prioritizes Problems. Chicago: University of Chicago Press.

King, Gary, Robert O. Keohane y Sidney Verba. 2000. El diseño de la investigación social. La inferencia cientifica en los estudios cualitativos. Madrid: Alianza Editorial.

Lascoumes, Pierre y Patrick Le Gàles. 2012. Sociologie de l'action publique (2. ${ }^{a}$ ed.). París: Armand Colin.

Lasswell, Harold. D. 1951. "The Policy Orientation», en Daniel Lerner y Harold D. Lasswell (eds.), The Policy Sciences: Recent Developments in Scope and Method. Palo Alto: Stanford University Press.

Lowndes, Vivien. 2019. "How Are Political Institutions Gendered?», Political Studies, 68 (3): 543-564. Disponible en: https://doi.org/10.1177/0032321719 867667.

Mackie, John L. 1965. "Causes and Conditions», American Philosophical Quarterly, 2 (4): 245-264. Disponible en: https://www.jstor.org/stable/20009173.

Mahoney, James. 2000. "Path Dependence in Historical Sociology», Theory and Society, 29 (4): 507-548. Disponible en: http://www.jstor.org/stable/3108585.

Mahoney, James y Celso Villegas. 2007. «Historical Enquiry and Comparative Politics», en Carles Boix y Susan C. Stokes (eds.), The Oxford Handbook of Comparative Politics, Oxford: Oxford University Press.

Majone, Giandomenico. 1997. Evidencia, argumentación y persuasión en la formulación de politicas. México DF: Fondo de Cultura Económica.

March, James G. y Johan P. Olsen. 1984. "The New Institutionalism: Organizational Factors in Political Life», The American Political Science Review, 78 (3): 734-749. Disponible en: https://doi.org/10.2307/1961840.

March, James G. y Johan P. Olsen. 1989. Rediscovering institutions. The organizational basis of politics. New York: The Free Press.

March, James G. y Johan P. Olsen. 2009. «Elaborating the New Institutionalism», en Sarah A. Binder, R. A. W. Rhodes y Bert A. Rockman (eds.), The Oxford Handbook of Political Institutions. Oxford: Oxford University Press.

McArthur, Daniel. 2011. «Book Review: The Conduct of Inquiry in International Relations: The Philosophy of Science and its Implications for the Study of World 
Politics», Education and Culture, 27 (2): 97-100. Disponible en: https://doi. org/10.1353/eac.2011.0008.

Méndez, José Luis. 1999. "Estudio introductorio», en B. Guy Peters, La política de la burocracia. México DF: Fondo de Cultura Económica.

Muller, Pierre. 2006. Las politicas públicas. Bogotá: Universidad Externado de Colombia. Mumford, Stephen. 1998. Dispositions. Oxford: Oxford University Press.

Muñoz, Nelson F. 2011. Análisis neoinstitucional de la politica pública para la reforma del sistema de transporte de Bogotá — sistema integrado de transporte público sitp-en su aporte a la construcción del derecho a la ciudad y a la movilidad en Bogotá. Bogotá: Pontificia Universidad Javeriana.

Offe, Claus. 1996. "Designing Institutions in East European Transitions», en Robert E. Goodin (ed.), The Theory of Institutional Design. Nueva York: Cambridge University Press. Disponible en: https://doi.org/10.1017/CBO9780511558320.008.

Olejniczak, Karol, Sylwia Borkowska-Waszak, Anna Domaradzka-Widła y Yaerin Park. 2019. «Policy labs: the next frontier of policy design and evaluation?», Policy and Politics, 48 (1): 89-110. Disponible en: https://doi.org/10.1332/030557 319X15579230420108.

Olsen, Johan P. 2008. "Understanding Institutions and Logics of Appropriateness: Introductory Essay», en James G. March (eds.), Understanding Organizations. Stanford: Stanford University Press.

Ortega-Bolaños, Jesús A. y Margarita I. Alba-Muñoz. 2017. «Discursos interpretativos y prácticas deliberativas: Propuesta metodológica para formulación de políticas públicas sanitarias en Colombia», Revista de Salud Pública, 19 (3): 386-392. Disponible en: http://dx.doi.org/10.15446/rsap.v19n3.67269.

Ostrom, Elinor. 2007. «Elección racional institucional. Evaluación del marco del análisis y desarrollo institucional», en Paul Sabatier (ed.), Teorías del proceso de las politicas públicas. Boulder, Colorado: Westview Press.

Ostrom, Elinor. 2014. «Más allá de los mercados y los Estados: gobernanza policéntrica de sistemas económicos complejos", Revista Mexicana de Sociología, 76 (SPE): 15-70. Disponible en: https://cutt.ly/TmxfvLY.

Parsons, Talcott. 1961. An Outline of the Social System. San Juan: University of Puerto Rico.

Peters, B. Guy. 2003. El nuevo institucionalismo: Teoría institucional en ciencia politica. Barcelona: Gedisa.

Peters, B. Guy y Guillaume Fontaine. 2020. «Introduction», en B. Guy Peters y Guillaume Fontaine (eds.), Handbook of Research Methods and Applications in Comparative Policy Analysis. Cheltenham: Edward Elgar.

Peters, B. Guy y Jon Pierre. 2005. «¿Por qué ahora el interés por la gobernanza?», en Agustí Cerrillo, La gobernanza hoy: 10 textos de referencia. Madrid: INAP.

Peters, B. Guy y Jon Pierre. 2020. «A typology of populism: understanding the different forms of populism and their implications", Democratization, 27 (6): 928-946. Disponible en: https://doi.org/10.1080/13510347.2020.1751615.

Popper, Karl R. 2008. La lógica de la investigación cientifica. Madrid: Tecnos. 
Rodrigues, Carlos. 2018. «Guillaume Fontaine. El análisis de políticas públicas en América Latina», Cuadernos del CENDES, 35 (97): 137-145.

Roe, Emery. 1994. Narrative Policy Analysis. Theory and Practice. Londres: Duke University Press. Disponible en: https://doi.org/10.1515/9780822381891.

Roth, André-Noël. 2014. Politicas públicas. Formulación, implementación y evaluación (10. ${ }^{a}$ ed.). Bogotá: Ediciones Aurora.

Rubio, Rocío y Alfredo Rosero. 2010. «El Advocacy Coalition Framework de Paul A. Sabatier: un marco de Análisis de Política Pública basado en coaliciones promotoras», en André-Noël Roth (ed.), Enfoques para el análisis de las políticas públicas. Bogotá: Universidad Nacional de Colombia.

Sabatier, Paul. 1988. "An Advocacy Coalition Model of Policy Change and the Role of Policy Orientated Learning Therein", Policy Sciences, 21 (2-3): 129-168. Disponible en: https://doi.org/10.1007/BF00136406.

Sabatier, Paul. 2010. Teorías del proceso de las políticas públicas. Buenos Aires: Jefatura de Gabinete de Ministros.

Sabatier, Paul y Christopher M. Weible. 2010. «El marco de las coaliciones promotoras. Innovaciones y aclaraciones», en Paul A. Sabatier (ed.), Teorías del proceso de las politicas públicas. Buenos Aires: Jefatura de Gabinete de Ministros.

Sartori, Giovanni. 1970. «Concept misinformation in comparative politics», American Political Science Review, 64 (4): 1033-1053. Disponible en: https://doi.org/ 10.2307/1958356.

Sayer, Andrew. 2010. Method in Social Science: A realist approach (2. ${ }^{\mathrm{a}}$ ed.). Nueva York: Routledge.

Scott, W. Richard. 2014. Institutions and organizations. Ideas, interests, and identities (4. ${ }^{\mathrm{a}}$ ed.). Washington DC: SAGE Publications.

Weber, Max. [1949] 2011. Methodology of social sciences. Nueva York: Routledge.

Wendt, Alexander. 1999. Social theory of international politics. Cambridge: Cambridge University Press.

Yanow, Dvora y Peri Schwartz-Shea. 2006. Interpretation and method: Empirical research methods and the interpretive turn. Nueva York: M. E. Sharpe.

Zabala-Peñafiel, Tania. 2017. «Analysis of the Ecuadorian Higher Education Reform, period 2008-2013, considering Public Policy Instruments», Journal of Education and Social Policy, 4 (1): 54-61.

Presentado para evaluación: 4 de junio de 2020.

Aceptado para publicación: 21 de mayo de 2021.

\section{ALEJANDRO HERNÁNDEZ-LUIS}

oahernandezf@@llacso.edu.ec

Doctorando en Políticas Públicas en la Facultad Latinoamericana de Ciencias Sociales, (FLACSO-Ecuador). Cuenta con una maestría en Políticas Públicas por la misma 
institución. Es investigador asociado del Laboratorio de Políticas Públicas Comparadas en el proyecto sobre "Controles Democráticos en América Latina». Investiga sobre diseño institucional y diseño de políticas anticorrupción.

\section{CAMILA CARRASCO}

camila.carrasco@uct.cl

Magíster en investigación de Políticas Públicas de la Facultad Latinoamericana de Ciencias Sociales (FLACSO-Ecuador). Administradora pública de la Universidad de Santiago de Chile. Actualmente trabaja en el Centro de Políticas Públicas de la Universidad Católica de Temuco e investiga sobre diseño de políticas públicas ambientales y controles democráticos en América Latina.

\section{SARAI GARCÍA-GUERRA}

saaraisofia93@gmail.com

Maestrante del programa de Investigación en Políticas Públicas de la Facultad Latinoamericana de Ciencias Sociales (FLACSO-Ecuador). Estudiante de la maestría en Psicología Clínica con Mención en Psicoanálisis y Psicopatologías. Es psicóloga, con mención en Psicología Social y Comunitaria. Su área de investigación principal son las políticas públicas de salud mental. 